\title{
High time resolution, two-dimensional position sensitive MSMGRPC for high energy physics experiments
}

\section{Petriş*! D.Bartoş, M. Petrovici, L. Rădulescu, V. Simion}

National Institute for Physics and Nuclear Engineering (IFIN-HH), Bucharest, Romania

Hadron Physics Department

E-mail: mpetris@nipne.ro

\section{J. Frünhauf}

GSI Helmholtz Center for Heavy Ion Research GmbH (GSI), Darmstadt, Germany

\section{Deppner, N. Herrmann}

Physikalisches Institut der Universität Heidelberg, Heidelberg, Germany

\begin{abstract}
Latest results of the R\&D activity devoted to the development of the multi-strip multi-gap resistive plate counter (MSMGRPC) prototype with the highest granularity of the Time of Flight (TOF) subsystem of the Compressed Baryonic Matter (CBM) experiment are reported in this contribution. The new designed MSMGRPC fulfills simultaneously the granularity requirement and the optimum matching of the characteristic impedance of the signal transmission line to the input impedance of the front-end electronics, exploiting the special architecture of the high voltage and signal electrodes. The results in terms of the efficiency and time resolution, obtained in the laboratory cosmic ray test using a triggered data acquisition system and in heavy ion in-beam test based on a free streaming readout in mCBM setup at SIS18/GSI Darmstadt, are reported.
\end{abstract}

European Physical Society Conference on High Energy Physics - EPS-HEP2019 -

10-17 July, 2019

Ghent, Belgium

\footnotetext{
*Speaker.

${ }^{\dagger}$ We acknowledge the financial support of projects RO-FAIR F04 and PN9060103.
} 


\section{Introduction}

Multigap Resistive Plate Chambers (MRPCs) [1] proving a very good time resolution and high efficiency at affordable costs were implemented in the time of flight (TOF) subsystems of large scale experimental set-ups, i.e HADES [2], FOPI [3], STAR [4], ALICE [5]. However, being based on resistive electrodes made of commercial float glass (with a resistivity of the order of $10^{12}-10^{13} \Omega \mathrm{cm}$ ), their rate capability does not exceed $\sim 1 \mathrm{kHz} / \mathrm{cm}^{2}$.

The next generation experiments, as is Compressed Baryonic Matter (CBM) experiment at the future experimental Facility for Antiprotons and Ion Research (FAIR) in Darmstadt, Germany, aims to investigate the QCD phase diagram in the region of high net-baryon densities and moderate temperatures, at interaction rates up to $10^{7}$ interaction/s [6]. In order to cope with such very high interaction rates, CBM will be equipped with fast and radiation hard detectors operated by a selftriggered readout electronics. The signals will be processed by a free-streaming data acquisition system, whereby all signals passing the electronic threshold will be digitized, time stamped and processed. The experimental setup includes a Time of Flight (TOF) wall [7] based on MRPCs with multi-strip readout [8] for hadron identification in fixed-target heavy ion collisions at SIS100 energies, up to $14 \mathrm{~A} \mathrm{GeV}$. The challenge for the TOF subsystem is to keep an efficiency larger than $95 \%$ and a system time resolution better than $80 \mathrm{ps}$ at high particle flux (up to $30 \mathrm{kHz} / \mathrm{cm}^{2}$ ) and hit multiplicity (up to 1000 tracks/event). For occupancy value below 5\%, the effective cell size of the TOF wall is scaled with the track density (which drops from low to large polar angles).

Therefore, we have focused on an intense R\&D activity $[9,10,11]$ for development of a Multi-Gap Resistive Plate Chamber with differential Multi-Strip readout (MSMGRPC) for the most demanding region of the TOF wall, the region of low polar angles $\left(2.5^{0}-11^{0}\right)$ called inner zone. We report in this paper latest results of the activity devoted to develop a MSMGRPC prototype with the highest granularity of the CBM-TOF wall. The counter fulfills simultaneously the granularity requirement and the optimum matching of the characteristic impedance of the signal transmission line impedance to the input impedance of the front-end electronics.

\section{MSMGRPC prototype with the highest granularity of the CBM-TOF wall}

\subsection{Inner geometry}

The MSMGRPC inner geometry is presented in Fig. 1. The typical architecture of our prototypes is a double stack structure, the six resistive glass plates of each stack being separated by five gaps of $140 \mu \mathrm{m}$ size. In order to cope with the high particle flux (see Sect. 1), the resistive electrodes are made of a special, new developed low resistivity glass [12], with an average resistivity of $1.5 \times 10^{10} \Omega \mathrm{cm}$. The outermost glass plates of each stack are in contact with the $\mathrm{Cu}$ strips of the high voltage electrodes for both positive and negative polarities. The overlaying readout electrodes are electrically isolated by the high voltage electrodes by thin insulator layers in order to prevent any possible discharges between the high voltage and readout electrodes. Two honeycomb plates positioned on the outer sides of the counter keep the alignment of the electrodes and the mechanical stability of the detector. The high voltage (HV) is distributed by a common high voltage line to each individual strip through $12 \mathrm{k} \Omega$ resistors which keep localized any possible perturbation of the potential of high voltage strips. 


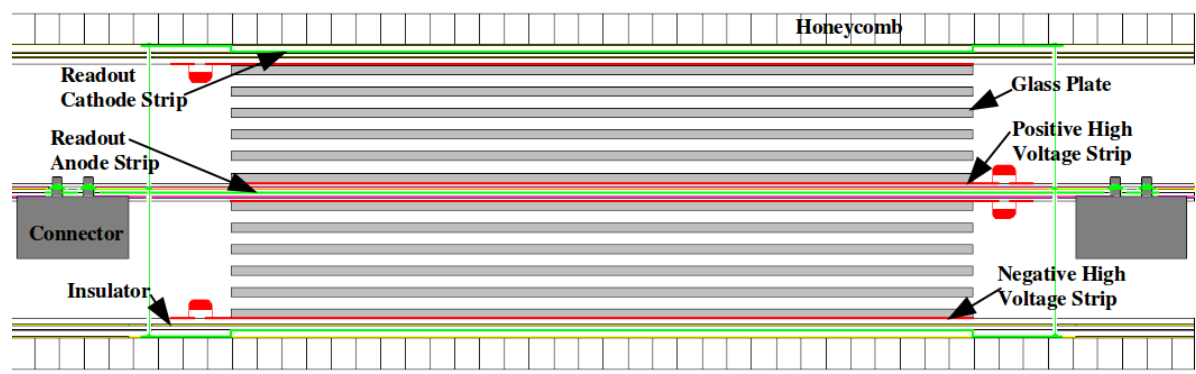

Figure 1: Cross section along the strip (green - readout strips, red - HV strips) .

\subsection{Readout electrodes and transmission line impedance}

The counter architecture has a strip structure for both HV and readout electrodes, with the same pitch for both of them (however, their strip width (HV and readout) can differ). The anode and cathode strips overlapped in space together with the layers positioned between them define a signal transmission line.

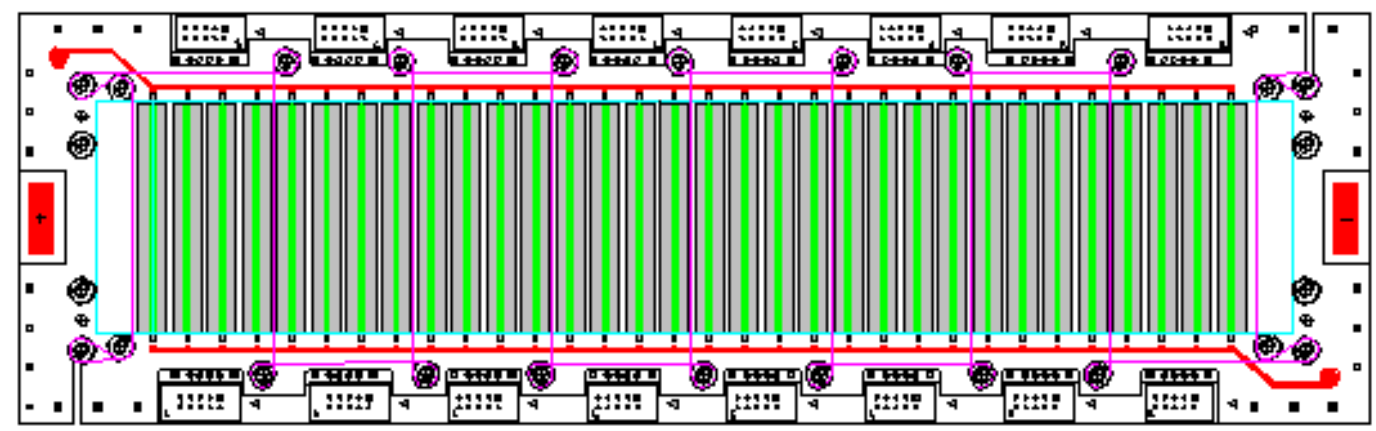

Figure 2: Front view of the prototype: green - readout strips, gray - HV strips, red - common HV line.

The $9.02 \mathrm{~mm}$ pitch and $60 \mathrm{~mm}$ length of the strips of the readout and high voltage electrodes have been chosen based on the maximum length $(300 \mathrm{~mm})$ of the available low resistivity glass and the occupancy requirement. The $7.37 \mathrm{~mm}$ high voltage strip width defines the granularity, taking into account the expected cluster size and the adjusted strip length. The width of the readout strip was estimated based on an innovative method described in reference [13]. For a given strip width of the HV electrodes, one could adjust the readout strip width in the signal propagation simulation using the software package APLAC [14], such to get a desired impedance which matches the input impedance of the front-end electronics. The values of the thickness and permitivity of the successive layers positioned between overlapped anode and cathode strips are considered in the simulations. A $1.27 \mathrm{~mm}$ readout strip width leads to a transmission line impedance of $97 \Omega$, which matches to the input impedance of the the front-end electronics channel. The alignment and overlap of the readout (green) and high voltage (gray) strips can be seen in the front view cross section shown in Fig. 2. On the up and down lateral sides are shown the high voltage lines (red) for positive and negative polarity. The signal connectors (black) are soldered on the lateral sides of the anode readout plate. The $300 \mathrm{~mm}$ maximum size of the low resistivity glass covered by 32 strips defines the length of the counter. The signals are readout in a differential mode, both the anode and 
the cathode readout strip signals being fed into the input of a front-end electronic channel. Each signal transmission line is readout at both ends.

\subsection{Prototype assembling}

Two identical prototypes were assembled (Fig. 3-left) and mounted one on top of the other in
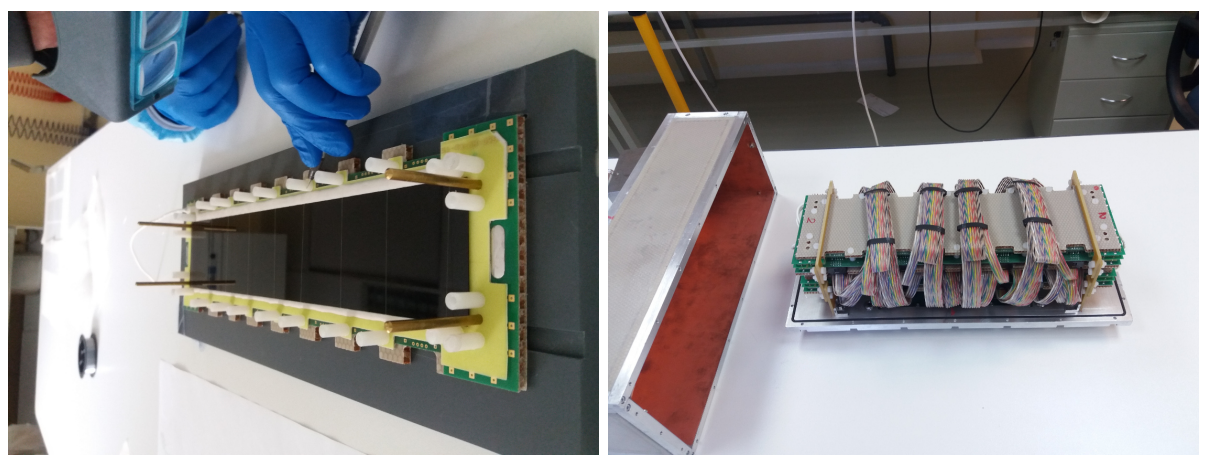

Figure 3: Spacer stretching across the resistive electrode - left. View of the assembled prototypes - right.

a gas tight and electromagnetically screened box, (Fig. 3 - right).

\section{Cosmic rays and in-beam tests}

\subsection{Cosmic rays tests}

The assembled counters were first tested in the detector laboratory using a gas mixture of $90 \% \mathrm{C}_{2} \mathrm{H}_{2} \mathrm{~F}_{4}+10 \% \mathrm{SF}_{6}$ and an electric field of $157 \mathrm{kV} / \mathrm{cm}(\mathrm{HV}= \pm 5.5 \mathrm{kV})$.

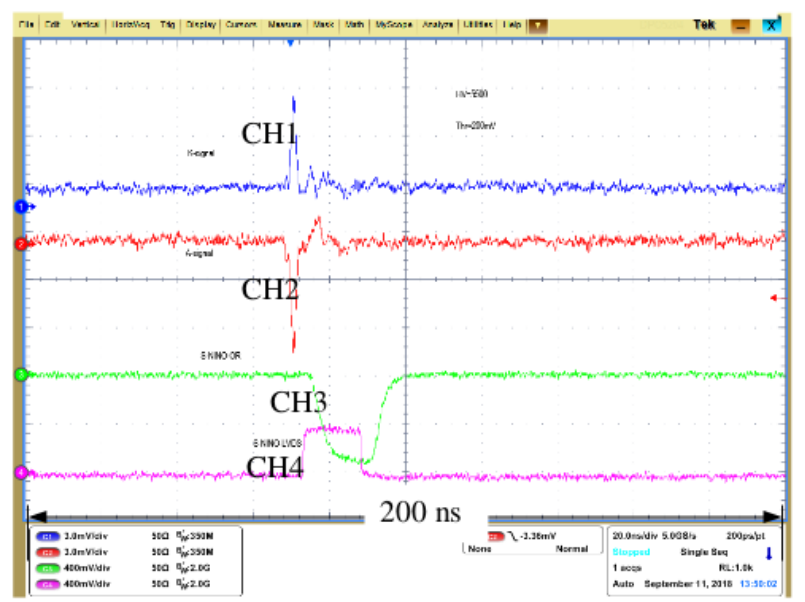

Figure 4: Cathode (blue - $\mathrm{CH} 1$ ) and anode (red - $\mathrm{CH} 2$ ) picked-up signals from one side of a transmission line, recorded directly on the oscilloscope, without any amplification. The logic OR signal (green - CH3) of the FEE card and the LVDS output of the NINO channel (magenta - CH4) are displayed.

The typical analog anode (red) and cathode (blue) signals recorded directly on Tektronix TDS 7254B $3 \mathrm{GHz}$ oscilloscope from one end of a signal transmission line are shown in Fig. 4, the other 
end being fed into the input of one of the 8 channels of NINO [15] based discriminator/amplifier, used as front-end electronics (FEE). As one can be seen, on a large time scale of 200 ns any reflection is displayed. On the same screenshot from Fig. 4, the logic OR signal (green) of the FEE card and the LVDS output of the NINO channel (magenta) were recorded.
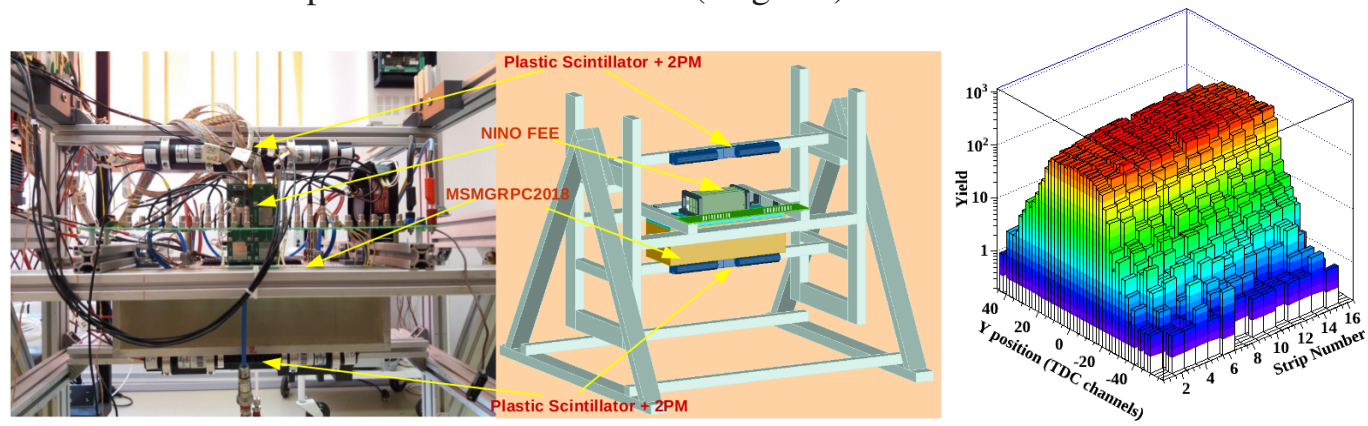

Figure 5: The cosmic ray experimental setup (left). 3D plot of the position of the cosmic ray events on the 16 measured strips (right).

In the experimental setup shown in Fig. 5 - left side, the two counters were sandwiched between two plastic scintillator bars of $10 \mathrm{~cm}$ length and $1.5 \mathrm{~cm} \times 1.5 \mathrm{~cm}$ cross section. The aim was to estimate the detector efficiency and time resolution before testing them in-beam, in real operation conditions. A triggered data acquisition (DAQ) system equipped with CAEN TDCs was used for signal processing. A 3D view of the cosmic-ray illumination across 16 operated strips per counter is shown in Fig. 5. For a $160 \mathrm{mV}$ FEE threshold the estimated efficiency was of $95 \%$ with an average cluster size of 1.8 strips. A single counter time resolution of $64 \pm 9$ ps was obtained after slewing corrections.

\subsection{In-beam tests}

The prototypes were tested in-beam in a dedicated CBM test setup called mCBM at the SIS18
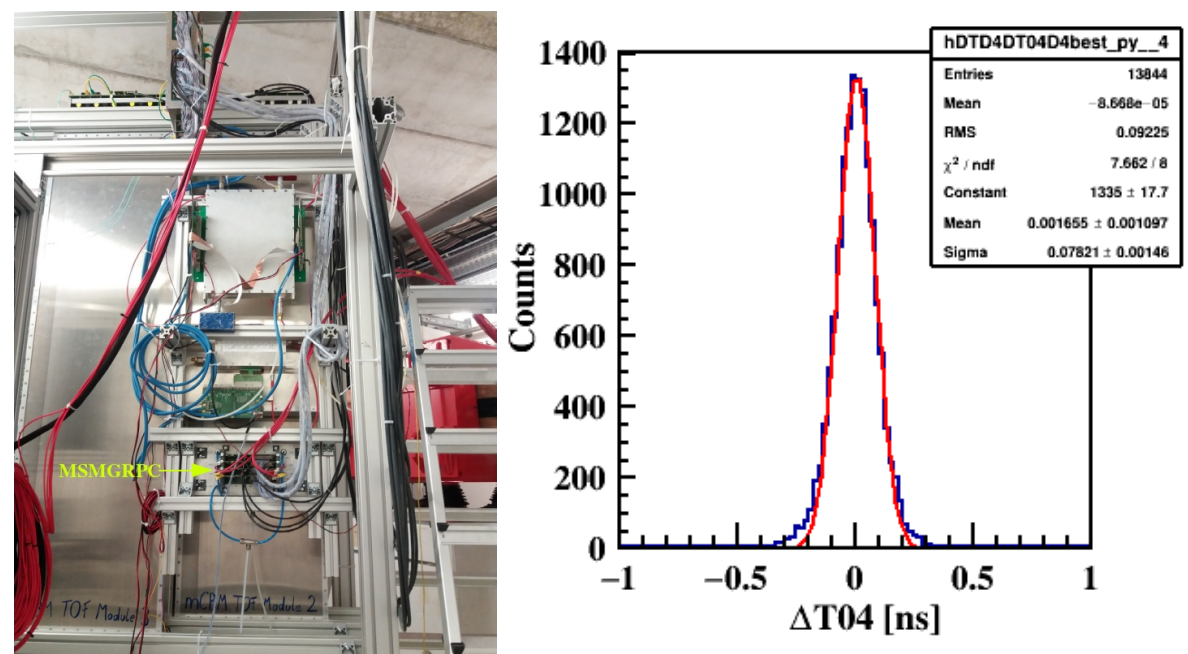

Figure 6: Position in the mCBM experimental setup (left). Calibrated time difference spectrum (right).

accelerator of GSI Darmstadt, with reaction products produced by $1.6 \mathrm{~A} \mathrm{GeV}{ }^{107} \mathrm{Ag}$ beam incident 
on a Au target. The above mentioned prototypes were positioned in the experimental setup downstream relative to the counters developed for the outer zones of the TOF wall (Fig. 6). A diamond detector, positioned in front of the target was used as beam reference. The signals delivered by the detectors were fed into PADIX [16] front-end electronics and processed by a free streaming DAQ equipped with GET4-TDCs [17]. For the estimation of the time resolution one of the two above described prototypes was considered as reference (REF) and the other one as detector under study (DUT). The obtained system time resolution (includes the contribution of both REF and DUT counters) was of $78 \mathrm{ps}$. If an equal contribution of the two identical counters to the time resolution is supposed, a single counter time resolution of $55 \mathrm{ps}$ (including electronics chain contribution) is estimated. The multi-dimensional data analysis is on-going and further results will follow.

\section{Conclusions}

A prototype of the counters with the highest granularity of the CBM-TOF wall was designed with the signal transmission line impedance matched to the input impedance of the front-end electronics. The cosmic ray test showed that the counter is free of reflections and has a very good performance in terms of efficiency and time resolution. The performance of the counter is confirmed by the preliminary results obtained in close to real conditions operation in the mCBM setup installed at SIS18, GSI Darmstadt.

\section{References}

[1] E. Cerron Zeballos et al., Nucl. Instrum. Meth. A, 374 (1996) 132.

[2] D. Belver et al., Nucl. Instrum. Meth. A, 602 (2009) 687.

[3] FOPI COllaboration, Nucl. Instrum. Meth. A, 646 (2011) 27.

[4] STAR Collaboration, Nucl. Instrum. Meth. A, 661, Suppl.1, (2012) s110.

[5] ALICE Collaboration, International Journal of Modern Physics A 29 (24) (2014) 1430044

[6] CBM Collaboration, Eur. Phys. J. A, 53 (2017) 60

[7] CBM Collaboration, CBM-TOF TDR (2014), GSI-2015-01999.

[8] M. Petrovici et al., Nucl. Instrum. Meth. A, 487 (2002) 337.

[9] M. Petrovici et al., JINST 7 P11003 (2012).

[10] M. Petriş et al., JINST 11 C09009 (2016).

[11] M. Petriş et al., Nucl. Instrum. Meth. A, 920 (2019) 100.

[12] J. Wang et al., Nucl. Instrum. Meth. A,713 (2013) 40.

[13] D. Bartoş et al., Rom.J. of Phys. 63 (2018) 901.

[14] https://www.rfglobalnet.com/doc/aplac-800-student-version-0001

[15] F. Anghinolfi et al., Nucl. Instrum. Meth. A, 533 (2004) 183.

[16] M. Ciobanu et al., IEEE Trans. Nucl. Sci. 61 (2014) 1015

[17] https://wiki.gsi.de/pub/EE/GeT4/get4.pdf 